Overview of the second stage in the comprehensive mirrors test in JET

This article has been downloaded from IOPscience. Please scroll down to see the full text article.

2011 Phys. Scr. 2011014070

(http://iopscience.iop.org/1402-4896/2011/T145/014070)

View the table of contents for this issue, or go to the journal homepage for more

Download details:

IP Address: 130.237.45.102

The article was downloaded on 27/01/2012 at 10:27

Please note that terms and conditions apply. 


\title{
Overview of the second stage in the comprehensive mirrors test in JET
}

\author{
M Rubel ${ }^{1}$, D Ivanova ${ }^{1}$, J P Coad ${ }^{2}$, G De Temmerman ${ }^{3}$, J Likonen ${ }^{4}$,

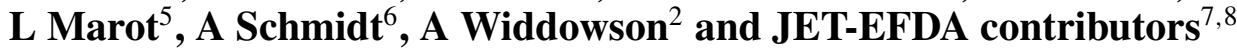 \\ ${ }^{1}$ Alfvén Laboratory, Royal Institute of Technology, Association EURATOM-VR, 10044 Stockholm, \\ Sweden \\ ${ }^{2}$ CCFE/EURATOM Fusion Association, Culham Science Centre, Abingdon OX14 3DB, UK \\ ${ }^{3}$ FOM Institute for Plasma Physics, Association EURATOM-FOM, Rijnhuisen, \\ NL-3439 MN Nieuwegein, The Netherlands \\ ${ }^{4}$ Association EURATOM-TEKES, VTT, PO Box 1000, 02044 VTT, Espoo, Finland \\ ${ }^{5}$ Department of Physics, University of Basel, Association EURATOM-CRPP, Basel, Switzerland \\ ${ }^{6}$ Institute for Energy and Climate Research-2, Forschungszentrum Jülich, Association EURATOM-FZJ, \\ Jülich, Germany \\ ${ }^{7}$ JET-EFDA, Culham Science Centre, Abingdon OX14 3DB, UK \\ E-mail: rubel@kth.se
}

Received 10 May 2011

Accepted for publication 22 July 2011

Published 16 December 2011

Online at stacks.iop.org/PhysScr/T145/014070

\begin{abstract}
The first mirror test for ITER in JET with carbon walls has been completed. Thirty polycrystalline Mo mirrors including four coated with a $1 \mu \mathrm{m}$ rhodium $(\mathrm{Rh})$ film were exposed to plasma in the divertor region and in the main chamber. The mirrors were installed in eight cassettes of pan-pipe shape. The reflectivity of all mirrors exposed in the divertor has been degraded by $80-90 \%$ because of the formation of thick $(>20 \mu \mathrm{m})$ flaking co-deposits on surfaces. Only small reflectivity losses (5-10\%) occurred on mirrors located at the channel mouth of the cassettes from the main chamber wall. This is due to the in situ removal of deposited species by charge exchange neutrals. Deuterium, ${ }^{12} \mathrm{C}$ and ${ }^{9} \mathrm{Be}$ are the main isotopes detected on surfaces, but other isotopes $\left({ }^{13} \mathrm{C}\right)$ are also found in some locations, thus indicating differences in the material migration. Rhodium coatings with an initial reflectivity that is $30 \%$ better than that of pure Mo survived the test without detachment, but their resultant reflectivity was the same as that of the exposed Mo surfaces.
\end{abstract}

PACS numbers: 52.55.Fa, 52.40.Hf, 52.25.Vy, 52.70.Kz

(Some figures may appear in colour only in the online journal)

\section{Introduction}

Metallic mirrors will be essential components of all optical systems for plasma diagnosis and imaging in a reactor-class device. Any degradation of the mirror reflectivity would influence the quality of signals that are crucial, in many cases, for the machine control. One has to recognize and assess the impact of the long-term exposure in a tokamak on the reflectivity change. Therefore, on the request of the ITER design team, the first mirror test (FMT) has been carried

\footnotetext{
${ }^{8}$ See the appendix of Romanelli F et al (2010) Proc. 23rd Int. Conf. on Fusion Energy (Daejon, Korea).
}

out at JET [1, 2]. The entire research program comprises: (a) selection of material for the test mirror, (b) manufacture of mirrors and their carriers (cassettes of pan-pipe shape) for in-vessel installation, (c) optical pre-characterization of mirrors, (d) exposure in the plasma boundary of JET during a complete operational campaign, (e) a broad range of post-exposure analyses by means of optical and surface analysis methods, (f) analyses of deposition in the channels of the mirror carriers [3] and (g) photonic cleaning of the exposed mirrors [4], followed by analyses of cleaned surfaces and examination of products generated by the laser light impact. The FMT is embedded in tritium retention studies [5] 
aiming at a detailed description of erosion-deposition patterns in JET measured by several types of diagnostic tools. These are located in the vicinity of the mirrors. It has been the most comprehensive test so far, performed with a large number of specimens, ITER-relevant materials for mirrors and long-term exposure to plasma. Two campaigns in JET with carbon walls have been performed. The changes in optical properties of mirrors retrieved from the vessel after the first campaign (2005-2007) have been discussed earlier [2]. The aim of the present work is to provide an overview of the results obtained during the second round of the test and, based on the outcome of the two campaigns, to discuss options for mirror maintenance and cleaning in a steady-state reactor.

\section{Experimental details: exposure and analyses}

Until now, two exposures have been performed in JET with carbon walls: step 1 in 2005-2007 comprising $126600 \mathrm{~s}$ $(35 \mathrm{~h})$ of plasma operation including $96900 \mathrm{~s}(26.9 \mathrm{~h})$ of $\mathrm{X}$-point operation [2] and the recently accomplished step 2 (2008-2009) with $161680 \mathrm{~s}$ (45h) exposure including $117680 \mathrm{~s}(32.7 \mathrm{~h})$ of X-point plasma. These plasma exposure times, according to scaling by divertor fluxes, correspond to only a very few full-power ITER pulses [6]. One set of mirrors located in the inner divertor was facing discharges during both campaigns, i.e. $80 \mathrm{~h}$ (59.6 h X-point) of plasma in total. Mirrors were exposed to all operation scenarios realized in JET for several years. The test in 2008-2009 was performed with 32 mirrors made of polycrystalline molybdenum (Mo-poly) including four specimens coated with a $1 \mu \mathrm{m}$ thick layer of rhodium (Rh). The coating was obtained by magnetron sputtering, as described in [7, 8].

Mirrors were installed in carriers (eight cassettes with either three or five channels) placed on the outer wall and in the divertor: the inner leg, the outer leg and the base plate under the load bearing tile. Mirrors were located in the cassettes at different distances from the channel mouth: from 0 to $4.5 \mathrm{~cm}$, thus resulting in different aspect ratios (depth in the channel to mirror width). Exact information regarding the construction and location of the mirror carriers has been given earlier [1]. Only some figures of merit are invoked in the following: solid angle for particle bombardment $1 \times$ $10^{-3}-0.2 \mathrm{sr}$, which is in the range for the mirrors foreseen in ITER: $3 \times 10^{-5}-1.4 \mathrm{sr}$. Rhodium-coated mirrors were placed in four cassettes: one in each of the major locations, at a distance of $1.5 \mathrm{~cm}$ from the channel mouth. Some mirrors that were placed on the main chamber wall near the beryllium evaporator were protected by a magnetic shutter that was open only in the presence of the magnetic field.

Before and after exposure, mirrors underwent a detailed surface analysis using optical methods, ion beam and microscopy techniques. Total reflectivity was measured in the range 350-1700 nm using a photo-spectrometer (GetSpec) system complying with work procedures on materials retrieved from JET $[1,2]$, i.e. contaminated with beryllium and tritium [9-11]. The integrating sphere was located in the glove-box and connected by fiber optics with externally located photo-spectrometers. The second, not-contaminated sphere was used for the pre-characterization of the mirrors. Full optical characteristics of some specimens from the main
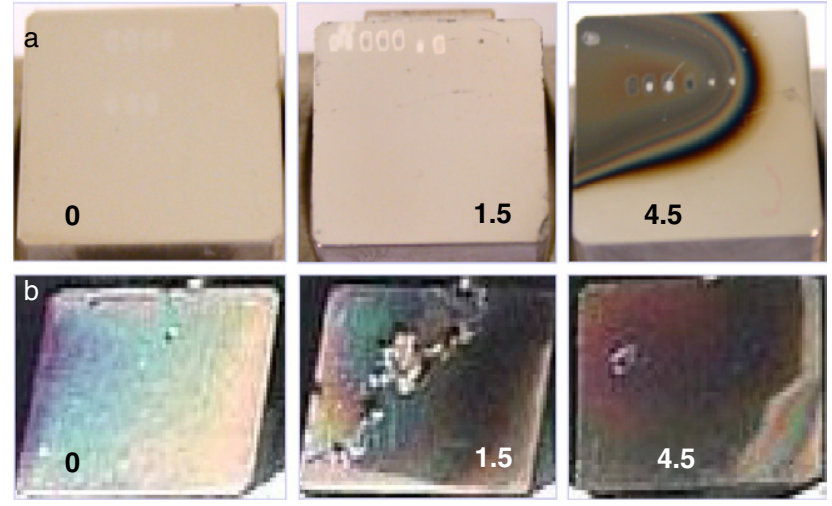

Figure 1. Mirrors retrieved after the exposure in 2008-2009 from (a) the main chamber wall and (b) the outer divertor. The numbers indicate the position in the channel, i.e. the distance in $\mathrm{cm}$ from the channel mouth. The spots seen on mirrors (a) are sputtered areas during SIMS. Mirrors at $1.5 \mathrm{~cm}$ are $\mathrm{Rh}$-coated; other specimens are pure Mo-poly.

chamber wall were recorded using a Varian Cary 5 apparatus working in the range $250-2500 \mathrm{~nm}$. In addition, the mirrors were characterized using a multiple angle of incidence spectroscopic ellipsometer (Sentech SE850) in the wavelength range $350-800 \mathrm{~nm}$ for incident angles $45^{\circ}, 55^{\circ}$ and $65^{\circ}$.

Surface and sub-surface composition was studied using ion and electron beam methods. Nuclear reaction analysis (NRA) with a $2.5 \mathrm{MeV}{ }^{3} \mathrm{He}^{+}$beam was used to quantify deuterium, carbon $\left({ }^{12} \mathrm{C}\right.$ and $\left.{ }^{13} \mathrm{C}\right)$ and beryllium by detecting protons emerging from the following reactions: ${ }^{3} \mathrm{He}(\mathrm{d}, \mathrm{p}){ }^{4} \mathrm{He}$, ${ }^{3} \mathrm{He}\left({ }^{12} \mathrm{C}, \mathrm{p}\right){ }^{14} \mathrm{~N}, \quad{ }^{3} \mathrm{He}\left({ }^{13} \mathrm{C}, \mathrm{p}\right){ }^{15} \mathrm{~N}$ and $\quad{ }^{3} \mathrm{He}\left({ }^{9} \mathrm{Be}, \mathrm{p}\right){ }^{11} \mathrm{~B}$. Carbon and beryllium were also determined by enhanced proton scattering (EPS) with a $2.5 \mathrm{MeV} \mathrm{H}^{+}$beam, which allowed simultaneous studies of heavier elements using proton-induced $\mathrm{x}$-ray emission (PIXE). Secondary ion mass spectrometry (SIMS) with a VG IX70S double-focusing magnetic sector equipment allowed depth profiling of hydrogen $\left({ }^{1} \mathrm{H}\right)$, deuterium $\left({ }^{2} \mathrm{H}\right)$, beryllium $\left({ }^{9} \mathrm{Be}\right)$, carbon $\left({ }^{12} \mathrm{C}\right)$, nickel $\left({ }^{58} \mathrm{Ni}\right)$, molybdenum $\left({ }^{98} \mathrm{Mo}\right)$ and rhodium $\left({ }^{103} \mathrm{Rh}\right)$. The analyses were performed using a primary oxygen beam $\left(\mathrm{O}_{2}^{+}, 5 \mathrm{keV}\right.$, ion current $\left.250 \mathrm{nA}\right)$. Sputtering at the rate of $0.35 \mathrm{~nm} \mathrm{~s}^{-1}$ was done on $300 \times 220 \mu \mathrm{m}^{2}$ areas. The topography was determined using scanning electron microscopy (SEM) with a field emission gun (Zeiss Gemini), whereas the composition was studied with energy dispersive X-ray (EDX) spectroscopy using an Oxford Instruments detector. X-ray photoelectron spectroscopy was used to study the material mixing on some mirrors from the main chamber wall. Chemical shift of the core levels Rh3d, Mo3d, O1s and C1s was determined.

\section{Results and discussion}

\subsection{Topography of the mirrors}

The series of photographs in figure 1 show the appearance of mirrors from the main chamber wall (a) and the outer divertor (b) after exposure in JET. The quality of images is somewhat obscured because of photographing through the plastic of the glove-box. The position of mirrors in cassettes is marked to inform about the distance in $\mathrm{cm}$ from the 

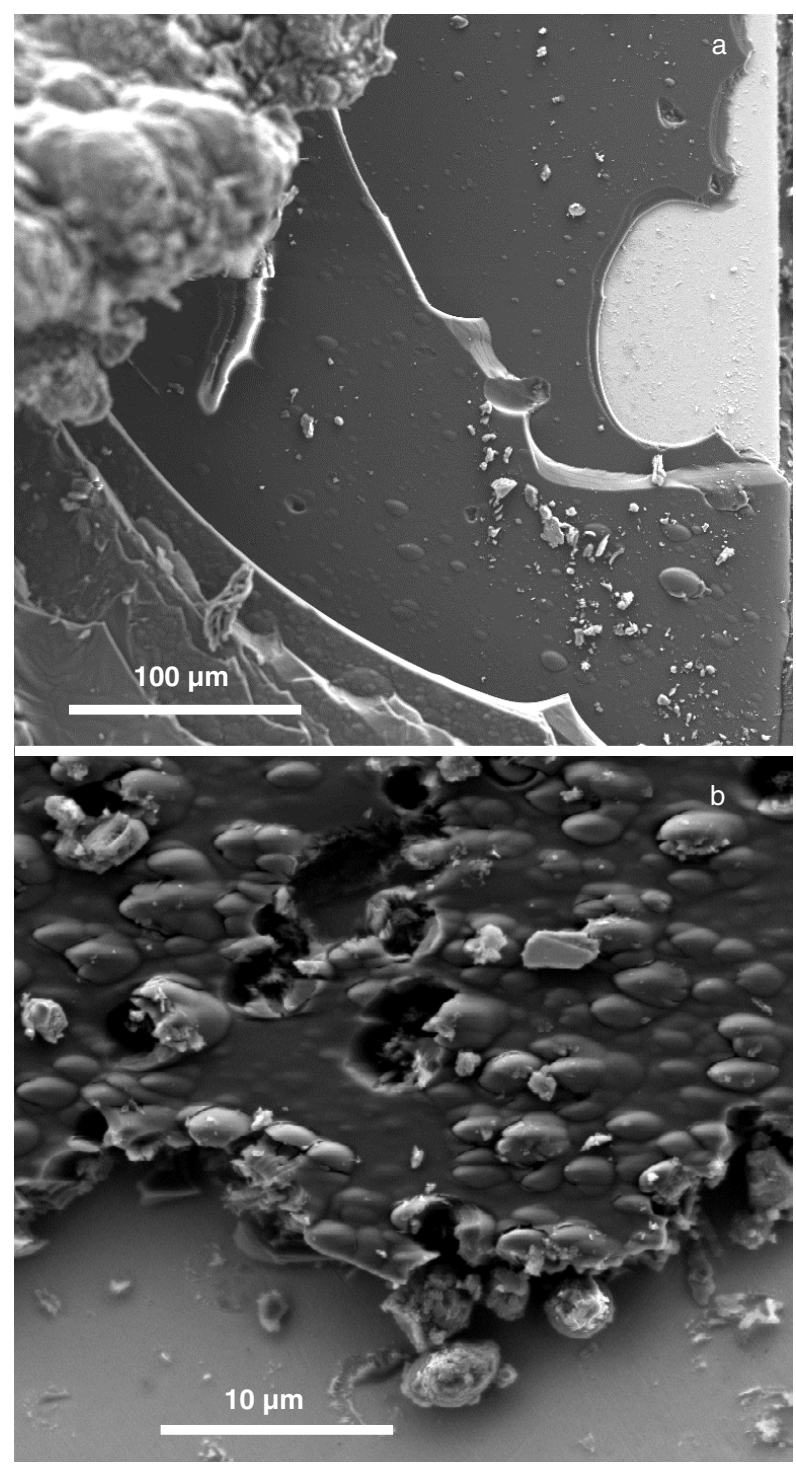

Figure 2. Surface topography of flaking co-deposits on mirrors from (a) the inner divertor (exposure at $0 \mathrm{~cm}$ during the 2005-2009 operation) and (b) the divertor base (exposure at $0 \mathrm{~cm}$ during the 2008-2009 campaign).

mouth of the channel. The bright areas on mirrors from the main chamber wall are spots eroded by sputtering during SIMS measurements. The images are representative of all the specimens from the two major locations inside the tokamak. Visual inspection indicates that (i) on the main chamber wall no deposit is perceived by eye on the mirrors close to the channel, but deposition is clearly seen on mirrors located deeper in the channel, i.e. 3.0 and $4.5 \mathrm{~cm}$, and (ii) all divertor mirrors are covered by deposits that are rather thick and flaking, and deposition decreases with depth in the channel. These results are in full agreement with findings after the first step of the mirror test in 2005-2007, as reported in [2, 3]. The results imply that the surface state of mirror in the divertor is governed by net deposition, whereas net erosion decides the state of mirrors located close to the channel mouth in cassettes on the main chamber wall. Micrographs in figures 2(a) and (b) show details of flaking co-deposits on surfaces exposed in the inner divertor $(0 \mathrm{~cm}, 2005-2009)$ and in the base (0 cm, 2008-2009), respectively. In figure 2(a), one observes a stratified structure of the layer, which peels-off, thus forming terraces and areas of diversified deposit thickness. Flaking and poor adherence to the substrate, as can be judged from figure 2(b), indicates that, during the entire period of exposure, many consecutive layers could peel off and contribute to the dust formation.

\subsection{Reflectivity of the exposed mirror}

Total reflectivity of all exposed mirrors was examined with spectro-photometry and compared with the initial parameters. The plots in figures 3(a) and (b) show the reflectivity of mirrors exposed in the inner divertor during the entire period of FMT (2005-2009) and during step 2 (2008-2009), respectively. The initial reflectivity of Mo-poly and Rh-coated mirrors is also shown in the graphs. Detailed data on the optical parameters of the mirrors (i.e. total, specular and diffuse reflectivity) have been presented in [1]; the diffuse reflectivity was on the level of $4-5 \%$. For clarity of presentation it is illustrated by a single plot, as the initial values for mirrors from a given category (either Mo-poly or Rh-coated) were very similar. The main result is that the reflectivity of nearly all mirrors in the divertor region has been degraded by $80-90 \%$. The loss of reflectivity is more pronounced in the visible range than in the infrared region. The results are very consistent with those obtained after the exposure in 2005-2007 [2, 3]. The strongest loss is measured on mirrors located close to the channel mouth. The exception for the trace at $0 \mathrm{~cm}$ in figure 3(b) is associated with the peeling-off of deposits. There is no difference between $\mathrm{Mo}$ and $\mathrm{Rh}$ on the divertor samples because degradation of optical performance is connected with deposition, which is not surface specific once the first layer of a carbon deposit is formed.

Total reflectivity of mirrors from the main chamber wall is shown in figures 4(a) and (b), where the results for units with and without a magnetic shutter are plotted, respectively. A clear general tendency is that the strongest reflectivity loss is on mirrors located deep in the channel ( 3 and $4.5 \mathrm{~cm}$ ), whereas only small changes are measured on Mo-poly mirrors located at the entrance $(0 \mathrm{~cm})$ and close to the channel mouth at $1.5 \mathrm{~cm}$. One may note even a slight improvement of reflectivity. This indicates that net erosion by impacting charge exchange $(\mathrm{CX})$ neutrals resulted in surface cleaning and, as a consequence, it could lead to a slight increase of reflectivity in the visible range. The recent results for Mo-poly fully confirm the tendency measured after the first test on mirrors retrieved in 2007 [2]. Rhodium-coated specimens were used in JET for the first time. The big initial difference in reflectivity between molybdenum and rhodium has not been maintained after the exposure. The values measured on Rh-coated and non-coated Mo mirrors are the same. In figure 5, there are data for specular reflectivity for surfaces exposed in the same wall unit at the channel mouth (pure $\mathrm{Mo}$ ) and $1.5 \mathrm{~cm}$ in the channel (Rh-coated). The plots nearly overlap in the entire spectral range, thus showing that the total and specular reflectivity have the same characteristics. It also confirms that more significant loss of performance occurred in the visible range than in the infrared region. Clarification of the possible reasons for the observed similarities of the 

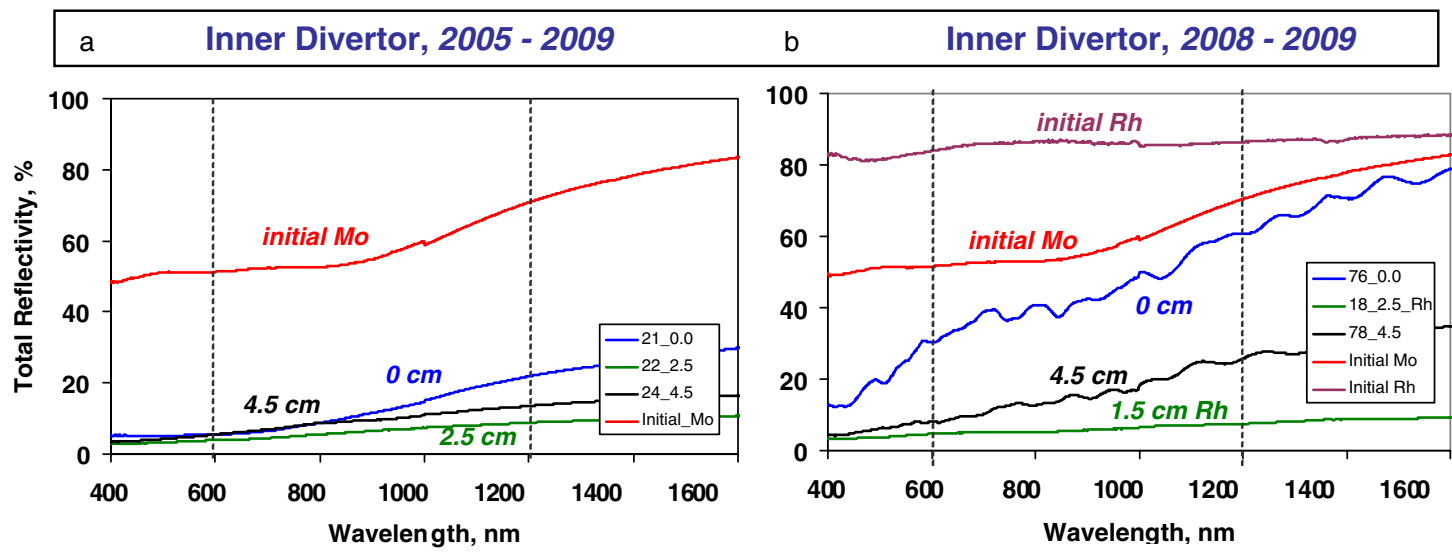

Figure 3. Total reflectivity of mirrors from the inner divertor after exposure: (a) 2005-2009 and (b) 2008-2009. An irregular trace for the position $0 \mathrm{~cm}$ in panel (b) is related to the peeling-off layer. Rhodium-coated mirrors are marked on the graphs; all other mirrors are pure Mo-poly.
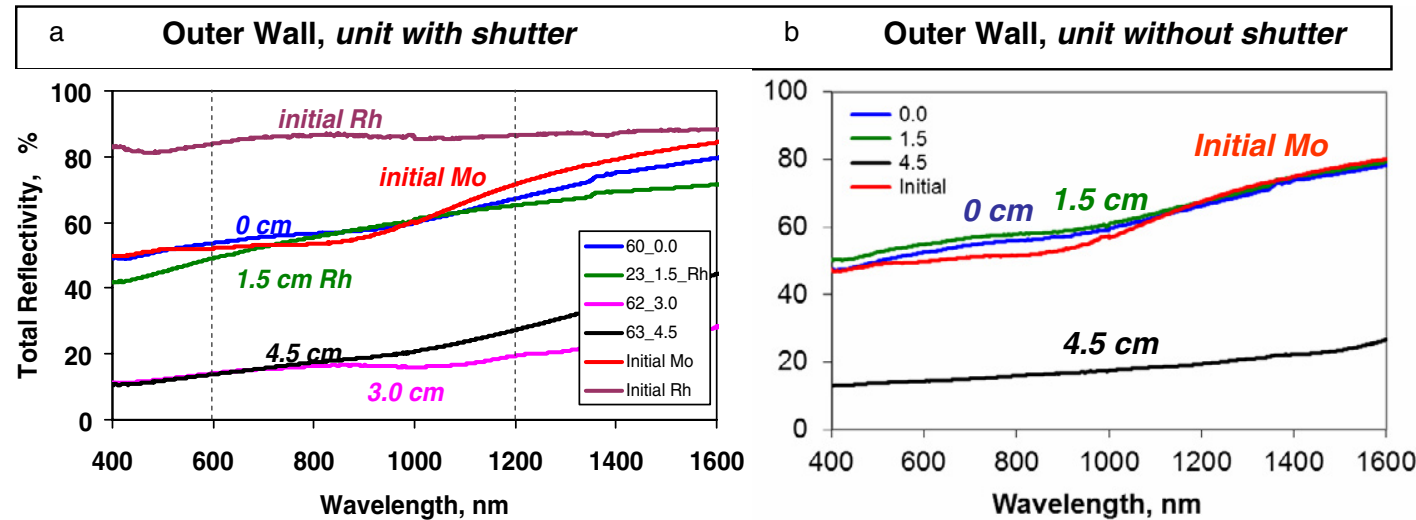

Figure 4. Total reflectivity of mirrors exposed in 2008-2009 on the outer wall in the main chamber: (a) the unit with a magnetic shutter; and (b) the unit without a shutter. Rhodium-coated mirrors are marked on the graphs; all other mirrors are pure Mo-poly.

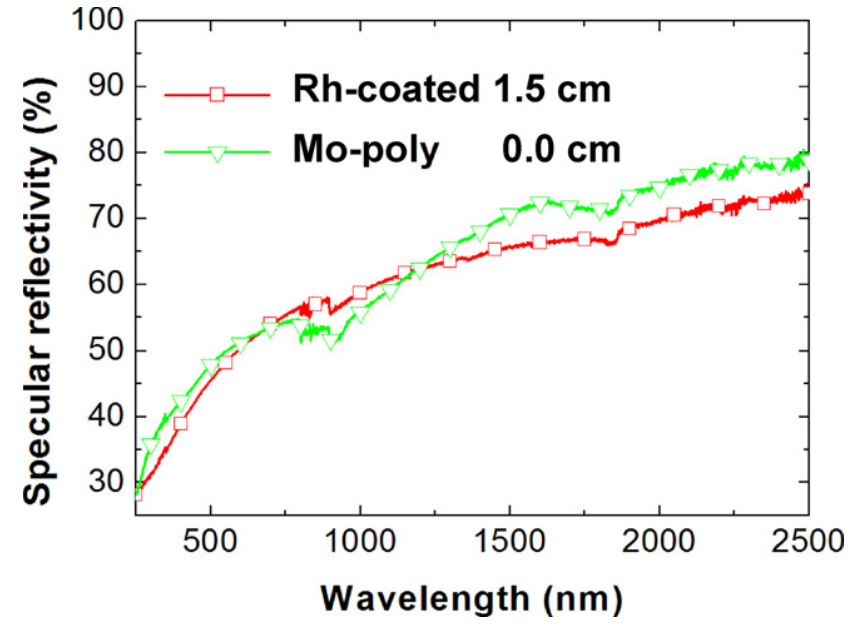

Figure 5. Specular reflectivity of Mo-poly and Rh-coated mirrors located close to the channel mouth in a cassette from the main chamber wall.

Mo-poly and Rh-coated surfaces (e.g. material mixing or Rh layer loss) has become an important point in further analyses.

\subsection{Surface composition of the exposed mirror}

NRA and EPS measurements combined with spectra modeling by means of the NDF program [12] revealed the presence of carbon-12 and deuterium on all the surfaces. The thickness of carbon-rich deposits on several studied surfaces was greater than the information depth with IBA: approximately $10 \mu \mathrm{m}$ with $2.5 \mathrm{MeV}^{3} \mathrm{He}^{+}$and up to $18 \mu \mathrm{m}$ with a $\mathrm{H}^{+}$beam. The graphs in figures $6(\mathrm{a})$ and (b) show carbon contents on mirrors from the divertor base and the outer wall, respectively. They also demonstrate the general tendency of specimens from the two major locations: (i) a decrease of deposition with depth in the channel in the divertor and (ii) a low deposition at the channel mouth and then an increase in deposition deeper in the channel on the wall. One may note that a similar amount of carbon was detected on the surfaces located deep in the divertor and at the channel mouth on the outer wall (about $1 \times 10^{17} \mathrm{~cm}^{-2}$ ). However, different phenomena could lead to this similarity. As demonstrated in figure 1, all mirrors from the divertor are coated with deposits, whereas no deposit is seen on surfaces located at 0 and $1.5 \mathrm{~cm}$ on the outer wall. This indicates that in the latter case, carbon may be implanted in the near surface region. The issue is discussed in the next section. For the outer wall mirrors, it is also perceived that the deposition first increases and then decreases slightly. This recent result is very consistent with previously obtained data [2], thus indicating that the CX flux reaching the surface is reduced because of a small solid angle and attenuation by collisions in the gas phase and with the channel walls. 


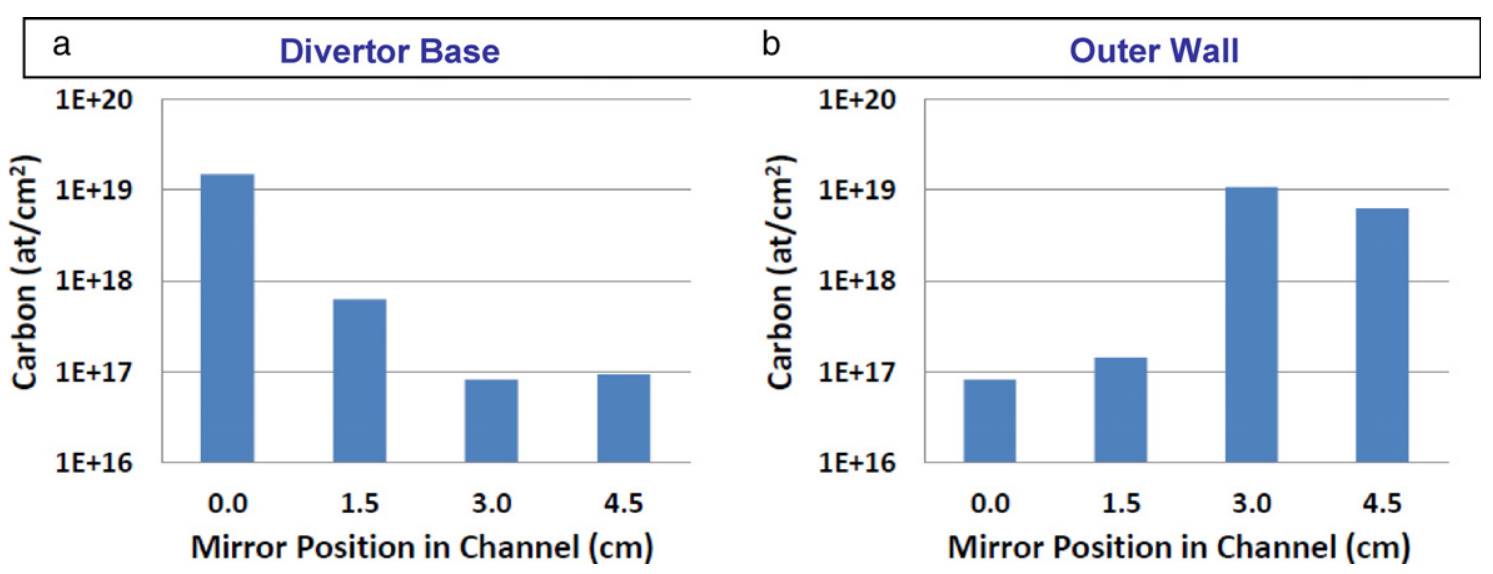

Figure 6. Carbon deposition on mirrors in (a) the divertor base and (b) the main chamber wall.

Other isotopes $\left({ }^{9} \mathrm{Be}\right.$ and $\left.{ }^{13} \mathrm{C}\right)$ are detected, but not on all the surfaces. Beryllium, in measurable amounts, is not found on surfaces from the inner divertor because of the limited transport of non-volatile species to that region, as discussed in $[10,11,13,14]$. Carbon-13 used as a tracer for material migration studies (as described in [14-16]) was puffed into JET on the last operation day of the campaign in 2009 from the outer divertor ring of gas inlet modules. The isotope was detected only locally, i.e. on the set of mirrors from the outer divertor: about $5.5 \times 10^{17}$ and $1.5 \times 10^{17} \mathrm{~cm}^{-2}$ on mirrors placed at a depth of 0 and $1.5 \mathrm{~cm}$, respectively. The amount of ${ }^{13} \mathrm{C}$ deposited during 30 discharges (about $460 \mathrm{~s}$ X-point) was compared with the amount of ${ }^{12} \mathrm{C}$ detected on mirrors after the entire exposure period $(161680 \mathrm{~s}): 1 \times 10^{18}$ and $3 \times 10^{17} \mathrm{~cm}^{-2}$ on the above-mentioned mirrors, thus giving a ${ }^{12} \mathrm{C} /{ }^{13} \mathrm{C}$ concentration ratio of about 2 . This small value is not proportional to the exposure time. One may deduce that this could be attributed to (i) quite frequent flaking and peeling-off of the carbon layers deposited on the mirrors in the divertor, (ii) the dynamic character of erosion and deposition in the divertor region, as discussed in [17].

\subsection{Modification of rhodium coating}

The aim of the analysis was to verify whether the $\mathrm{Rh}$ coating remained on the mirrors, especially on the specimen from the outer wall whose surface was strongly bombarded by CX neutrals. SEM-EDX and PIXE measurements have shown that a rhodium layer is present on all the four coated mirrors. The recorded intensity of the $\mathrm{Rh} \mathrm{K} \alpha$ x-ray line is clearly related to the amount of $\mathrm{C}$ and Be deposition on surfaces. A relatively weak $\mathrm{Rh} K \alpha$ signal has been recorded for the inner divertor mirror and the strongest from the outer wall specimen. No noticeable molybdenum $\mathrm{x}$-ray $\mathrm{K} \alpha$ signals have been detected (i.e. no Mo above the PIXE detection limit, 1 at. $\%$ ) on the Rh-coated mirrors, thus indicating (i) no detachment or peeling-off of the Rh film, (ii) no significant erosion of the coating, (iii) no Rh-Mo intermixing or diffusion because of the fairly low temperature of mirrors during the exposure: $220-280^{\circ} \mathrm{C}$ for cassettes on the outer wall and $170-200^{\circ} \mathrm{C}$ in the divertor. Plots in figure 7 are SIMS depth profiles of several isotopes on the Rh-coated mirror from the outer wall, i.e. the surface free from visible deposition. These results are qualitative (no quantitative data can be extracted),

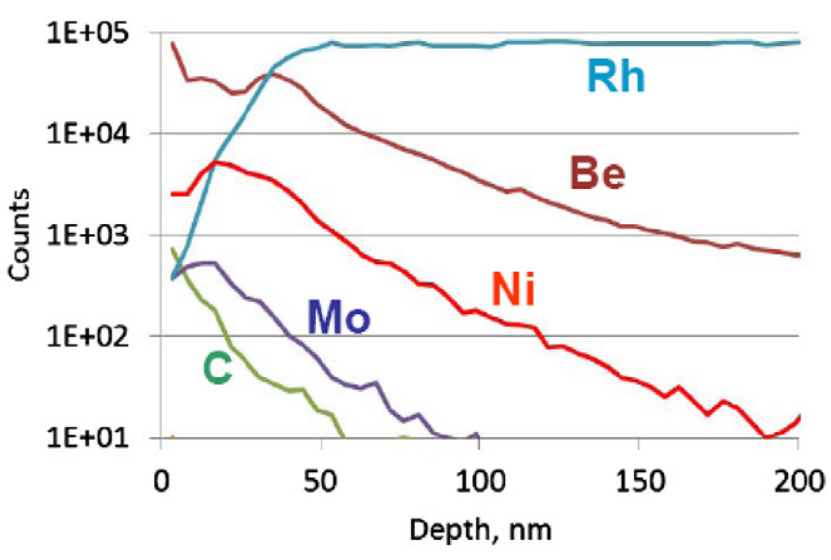

Figure 7. SIMS depth profiles of species in the surface region of a Rh-coated mirror exposed on the main chamber wall.

but they provide crucial information on the relative changes regarding the presence of respective species. The $\mathrm{Rh}$ coating extends to about $1 \mu \mathrm{m}$. It is in agreement with the nominal pre-exposure value and clearly proves that the film was not detached from the substrate. However, one may perceive that the near surface region up to $50 \mathrm{~nm}$ has a modified composition. In addition to carbon and beryllium, whose presence has been detected by NRA, there are also heavier species $\mathrm{Ni}$ and Mo, whose presence could be detected due to the very high sensitivity of SIMS. Their signals sharply decrease, indicating that the origin of the metals is not related to the erosion of the mirror substrate. Both elements originate from the erosion of the Inconel 625 alloy constituting the JET vacuum vessel. The alloy nominally contains up to $8-10 \mathrm{wt} \% \mathrm{Mo}$, and contains $58 \mathrm{wt} \% \mathrm{Ni}$ and $20-23 \mathrm{wt} \% \mathrm{Cr}$ as the main constituents. A detailed analysis of the profiles for the surface and sub-surface regions of two mirrors, Rh-coated and Mo-poly, exposed at the same depth in channels $(1.5 \mathrm{~cm})$ in two units on the main chamber wall clearly shows similarity in the overall surface composition: $\mathrm{C}, \mathrm{Be}, \mathrm{Mo}$ and $\mathrm{Ni}$, which were co-deposited and co-implanted as a result of the $\mathrm{CX}$ bombardment. This thin mixed material layer on the mirror surface is decisive for reflectivity. This may explain why the behavior of the Rh-coated and pure Mo-poly mirrors exposed under erosion-dominated conditions is eventually on the same level. 


\section{Concluding remarks}

The FMT in JET with carbon walls has been completed. The results of two long-term campaigns (nearly $40 \mathrm{~h}$ of plasma each) are very consistent: significant loss of optical performance in the divertor and smaller changes for mirrors on the outer wall. An important and positive result is that Mo-poly mirrors located at the channel mouth during exposure on the main chamber wall retained good reflectivity despite surface modification by material mixing. This may indicate the beneficial effects of net erosion of the Mo surface by CX neutrals. However, the same type of mixing was determined by SIMS on the Rh-coated mirror (from the same location), but its reflectivity dropped to the level measured for Mo specimens. It remains to be determined whether the surfaces contain just a mixture of co-implanted elements or a complex mixture of compounds. The fate of $\mathrm{Rh}$ coatings may be crucial in the final selection and qualification of mirror materials for ITER. It is clearly shown that the coatings survived the test. However, taking into account material mixing effects, these first results on long-term testing of the Rh layers do not provide convincing arguments for the application of coated mirrors in a reactor-class machine.

Optical performance of all mirrors in the divertor region has been fully degraded by deposition of carbon as the main constituent on the surfaces. It is not straightforward, and it is not intended here, to immediately translate these results to the ITER operation because of different densities and another wall composition. However, the results indicate that diagnostic mirrors in ITER may become coated with deposits in less than some tens of shots if carbon wall components are used. This result calls for intense efforts in the development of mirror replacement [2] or protection systems [18] even if solutions constitute a serious engineering challenge. The other option is the elaboration of reliable and feasible cleaning techniques to remove deposits and regain reflectivity. Whichever cleaning method is pursued the issue of dust formation is to be taken into account. The study has shown that deposits detach and peel off rather easily, as proven by visual inspection, microscopy and with a ${ }^{13} \mathrm{C}$ tracer, but this process occurs in an uncontrolled way. Dust mobilization in a diagnostic channel would additionally obscure optical signals. A certain type of mechanical cleaning is planned at JET.

However, what is most important is to test mirrors and other diagnostic components in a tokamak with metal walls where carbon transport would be suppressed. Therefore, FMT will be continued during JET operation with the ITER-like wall [19-21]. For that purpose, a new set of mirrors has been manufactured and installed.

\section{Acknowledgments}

This work, supported by the European Communities under the contract of Association between EURATOM/VR, was carried out within the framework of the European Fusion Development Agreement. The views and opinions expressed herein do not necessarily reflect those of the European Commission. This work was partly funded by the Swedish Research Council (VR) through contract no. 621-2009-4138.

Published under license from EURATOM.

\section{References}

[1] Rubel M et al 2006 Rev. Sci. Instrum. 77063501

[2] Rubel M et al 2009 J. Nucl. Mater. 390/391 1066

[3] Rubel M et al 2010 Nucl. Instrum. Methods A 623818

[4] Widdowson A et al 2011 J. Nucl. Mater. 415 S1199

[5] Coad J P et al 2005 Fusion Eng. Des. 74745

[6] Pitts R A et al 2005 Plasma Phys. Control. Fusion B 47300

[7] Marot L et al 2008 Thin Solid Films 5167604

[8] Marot L et al 2008 Surf. Coat. Technol. 2022837

[9] Andrew P et al 1999 Fusion Eng. Des. 47243

[10] Coad J P et al 2003 J. Nucl. Mater. 313-316 419

[11] Rubel M et al 2003 J. Nucl. Mater. 313-316 321

[12] Barradas N P et al 2007 Nucl. Instrum. Methods B 262282

[13] Coad J P et al 2001 J. Nucl. Mater. 290-293 224

[14] Coad J P et al 2006 Nucl. Fusion 46350

[15] Coad J P et al 2011 Phys. Scr. T145 014003

[16] Likonen J et al 2011 Phys. Scr. T145 014004

[17] Kreter A et al 2009 J. Nucl. Mater. 390/391 38

[18] Udintsev V S 2010 Nucl. Instrum. Methods A 623649

[19] Matthews G F et al 2007 Phys. Scr. T128 137

[20] Matthews G F et al 2009 Phys. Scr. T138 014030

[21] Matthews G F et al 2011 Phys. Scr. T145 014001 